\title{
Regional citrate anticoagulation for continuous renal replacement therapy without post-filter monitoring of ionized calcium
}

\author{
Tibor Fülöp ${ }^{1^{*}}$, Sohail Abdul Salim¹, Lajos Zsom ${ }^{2}$ \\ ${ }^{1}$ Department of Medicine, Division of Nephrology, University of Mississippi Medical Center, Jackson, MS, USA \\ ${ }^{2}$ Fresenius Medical Care Hungary Kft, Cegléd, Hungary
}

\section{A R T I C L E I N F O}

Article Type:

Review

\section{Article History:}

Received: 5 August 2017

Accepted: 4 November 2017

Published online: 13 December 2017

\section{Keywords:}

Continuous renal replacement therapy, Hemofiltration, Medical errors, Regional citrate anticoagulation, Thrombocytopenia, Intensive care unit

\begin{abstract}
A B S T RA C T
Continuous renal replacement therapy (CRRT) modalities are usually preferred in hemodynamically unstable patients in the intensive care units (ICU) but perceived expense and complexity slows broad acceptance. Heparin remains a problematic choice for CRRT anticoagulation due to the risk of bleeding in ICU patients and concerns about heparininduced thrombocytopenia. In this paper, we are describing our simplified regional citrate anticoagulation protocol, utilizing commercially available, premixed solutions exclusively and minimized laboratory monitoring. The protocol is employing Anticoagulant Citrate Dextrose-A (ACD-A) solution for citrate delivery, calcium-free dialysate or replacement fluids and separate calcium infusion, all commercially available in the United States. ACD-A is being infused pre-filter with an hourly rate of 1.5:1 to blood flow rate per minute without specific monitoring of post-filter ionized calcium concentration. Separate infusions of calcium-chloride, sodium phosphate and magnesium chloride are employed via triple lumen catheter to normalize peripheral ionized calcium, phosphate and magnesium concentrations, respectively. The protocol can be conveniently applied in both continuous veno-venous hemofiltration and hemodiafiltration regimens with several of the commercially available CRRT platforms. Built-in features of the protocol are the tendency alkalization and mild hypernatremia, which may be advantageous under select circumstances.
\end{abstract}

Implication for health policy/practice/research/medical education:

Establishing continuous renal replacement therapy (CRRT) in the intensive care units in a cost-effective manner remains a challenge. Regional citrate anticoagulation (RCA) during CRRT circumvents the risk of systemic anticoagulation, but represents yet another layer of complexity on an already intricate technology. Abandoning post-filter monitoring of ionized calcium during RCA offers improvement over existing approaches, including reduced complexity, potential for cost-saving and decreased potential for medical errors.

Please cite this paper as: Fülöp T, Abdul Salim S, Zsom L. Regional citrate anticoagulation for continuous renal replacement therapy without post-filter monitoring of ionized calcium. J Renal Inj Prev. 2018;7(3):139-143. doi: 10.15171/jrip.2018.35.

\section{Introduction}

Providing safe and reliable renal replacement therapy in the intensive care setting remains a challenge. While continuous renal replacement therapy (CRRT) modalities are usually preferred in hemodynamically unstable patients, difficulties pertaining to the complexity of such therapy exist. Many aspects of CRRT delivery remain vigorously debated, including the timing and indications of initiation, dose and duration of therapy and the choice of anticoagulants (1-4). Preventing clot formation is key for successful delivery of renal replacement support (5). Heparin, the agent historically used for anticoagulation of the extracorporeal circuit, may also induce thrombocytopenia (6) or increase the risk of bleeding in patients already at high risk. Further, the suppression of the platelet count is frequently observed in the intensive care unit (ICU) setting due to a multitude of reasons, including drug effects, excessive uptake and the presence of acute critical illness itself (7-9). Thus, not surprisingly, the search has been on for some time seeking

\footnotetext{
*Corresponding author: Tibor Fülöp, M.D., FACP, FASN, Division of Nephrology, University of Mississippi Medical Center, 2500 North State Street, L 504; Jackson, Mississippi 39216-4505. Present address of corresponding author: Department of Medicine - Division of Nephrology, Medical University of South Carolina, Charleston, USA, Email: tiborfulop.nephro@gmail.com
} 
an alternative option of achieving regional anticoagulation in the extracorporeal circuit.

\section{Materials and Methods}

We conducted a literature search on three databases including PubMed, EMBASE, Scopus and Google Scholar. The search was performed using a combination of the following terms; continuous renal replacement therapy, hemofiltration, regional citrate anticoagulation, thrombocytopenia and intensive care unit. Further, the authors' clinically experience was considered, when writing this protocol paper.

\section{Regional citrate anticoagulation}

Regional citrate anticoagulation (RCA) is an attractive candidate to achieve this goal (10-13). However, RCA may potentially add substantially to the complexity of an already complicated technology, impeding acceptance and utilizing excessive resources $(11,14)$. Delivering care in a uniform, safe and standardized setting with minimized need for laboratory testing appears to be the cornerstone of the implementation of RCA in the ICU setting. Simplifying the delivery of CRRT and offering uniform protocol may enhance acceptance by nursing staff and hospital administrators alike. In this paper, we would like to offer a description of a safe and effective standardized protocol with a potential to minimize errors.

\section{General concepts of regional citrate anticoagulation}

For an adult weighing $70 \mathrm{~kg}$, the total amount of dissolved calcium in the extracellular space is approximately 1000 $\mathrm{mg}$ at any given time. Under normal circumstances, total calcium concentration is approximately $2.5 \mathrm{mM} / \mathrm{L}$ (or $10 \mathrm{mg} / \mathrm{dL}$ ), of which about $50 \%$ is ionized, $13 \%$ is bound by small anions (lactate, citrate and phosphate) and the rest by negatively charged albumin molecules. This calcium amount is the approximate equivalent of $\sim 4$ amps of $10 \%$ calcium-chloride $(6.8 \mathrm{mM}$ of calcium per each $10 \mathrm{~mL}$ ) or $\sim 12$ amps of $10 \%$ calcium gluconate (93 $\mathrm{mg}$ of calcium or $2.3 \mathrm{mM}$ per each $10 \mathrm{~mL}$ ). At physiologic $\mathrm{P}^{\mathrm{H}}$ and ionic strength, ionized calcium is expected to decrease by $0.1 \mathrm{mM} / \mathrm{L}$ with each $0.5-0.6 \mathrm{mM} / \mathrm{L}(10 \mathrm{mg} /$ $\mathrm{dL})$ rise of plasma citrate. Assuming an initial ionized calcium concentration of 1.0-1.25 mM/L, ionized calcium is fully expected to be depleted once citrate concentration reaches about 5-6 mM/L (or $100 \mathrm{mg} / \mathrm{dL}$ ). At this point, similarly to the blood exposed to anticoagulant ethylenediaminetetraacetic acid (EDTA), the coagulation cascade is rendered ineffective and blood clot formation impaired. Citrate exposure is known to confer less filterinduced complement activation, neutrophil degranulation and less endothelial activation than heparin during continuous hemofiltration (15). To deliver a cheap and easily available form of citrate supplementation, the Anticoagulant Citrate Dextrose-A (ACD-A) solution is an obvious choice. It contains an isotonic mixture of citric acid $(0.8 \%)$, trisodium citrate $(2.2 \%)$ and dextrose, resulting in a final citrate concentration of $3.0 \%$ (or 112.9 $\mathrm{mM} / \mathrm{L}$ ). The normalization of systemic ionized calcium is achieved by simultaneous infusion of calcium-chloride and the endogenous metabolism of citrate, in the liver converting to bicarbonate in an approximate ratio of $1: 3$ and, simultaneously, releasing ionized calcium from the chelated form.

\section{Goals of therapy}

Successful implementation of CRRT should accommodate the following objectives;

- Utilization of physiologic or near-physiologic fluid solutions requiring little specific monitoring.

- Avoiding "customized" or pharmacy-made solutions, thus minimizing potential for medical errors.

- Avoiding systemic anticoagulants such as heparin in patients who are inherently at increased risk of bleeding.

- Minimizing clotting of extracorporeal circuit, thus avoiding frequent restarts and lost time with no ongoing renal replacement therapy.

- Avoiding the need for post-filter calcium monitoring, thus reducing the need for frequent testing and avoiding confusion resulting from markedly "abnormal" results of post-filter ionized calcium values.

In 2008, the nephrology faculty of our medical center revised the existing protocols of continuous venovenous hemofiltration (CVVHF) and hemodiafiltration (CVVHDF) regimens to deliver RCA in a uniform manner while minimizing cost, complexity and potential risk for the recipients of the therapy. We specifically desired the practice of abandoning post-filter monitoring of ionized calcium, highly inaccurate procedure during RCA. As we are a training institution regularly working with physicianin-training (nephrology fellows), it was important at that time to minimize handwritten orders to complement existing pre-printed order sets. Samples of our protocols are shown in Figures 1 and 2. These protocols can also easily be converted into electronic instruction formats in the current era.

As a rule, we routinely start with a blood flow of 200-250 $\mathrm{mL} / \mathrm{min}$ for our adult patients. ACD-A solution (available from multiple manufacturers) is infused pre-filter at a rate of $\times 1.5$ of the blood flow rate in $\mathrm{mL} / \mathrm{L}$ (e.g. for a blood flow rate of $200 \mathrm{~mL} / \mathrm{min}$ an $\mathrm{ACD}-\mathrm{A}$ infusion rate of 300 $\mathrm{mL} / \mathrm{h}$ will be applied). For replacement fluid or dialyzate fluid we are using calcium-free premixed solutions, close to physiologic concentrations (PrismaSate, PrismaSol; Gambro Renal Products Inc., Lakewood, CO), shown in Figures 1 and 2, second parts. The total effluent is calculated to achieve a net clearance of $>20-25 \mathrm{~mL} / \mathrm{kg} / \mathrm{h}$. In pure convective modality (CVVHF), we are routinely splitting the pre- and post-filter rate of the replacement fluid at 50:50 or 70:30 percent. A separate I.V. infusion will deliver calcium with calcium-chloride at an initial rate of $25 \mathrm{~g} / 24 \mathrm{~h}(500 \mathrm{~mL}$ bag containing $25 \mathrm{~g}$ of calcium chloride 

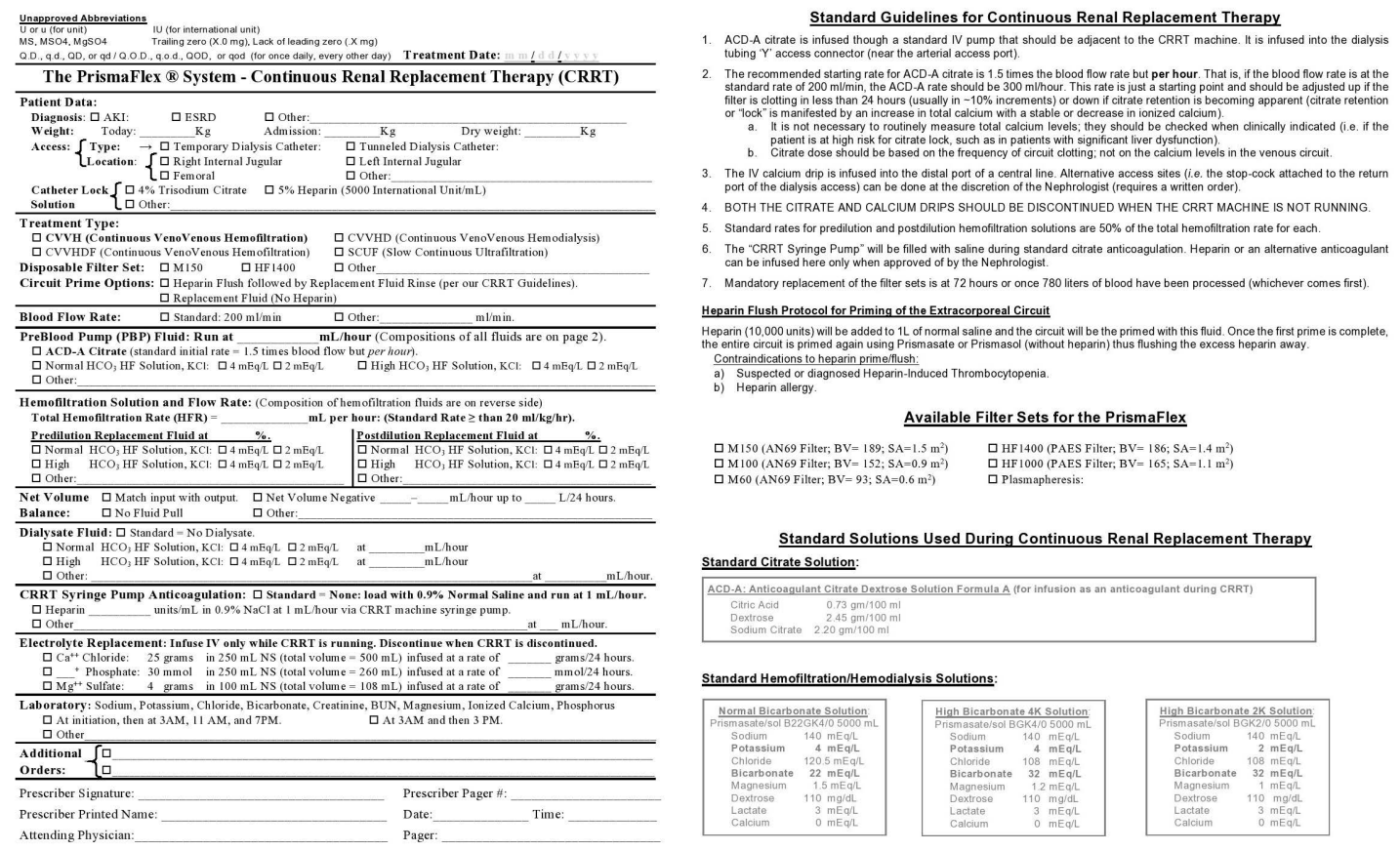

Figure 1. Adult Prismaflex CVVHD/HDF protocol.

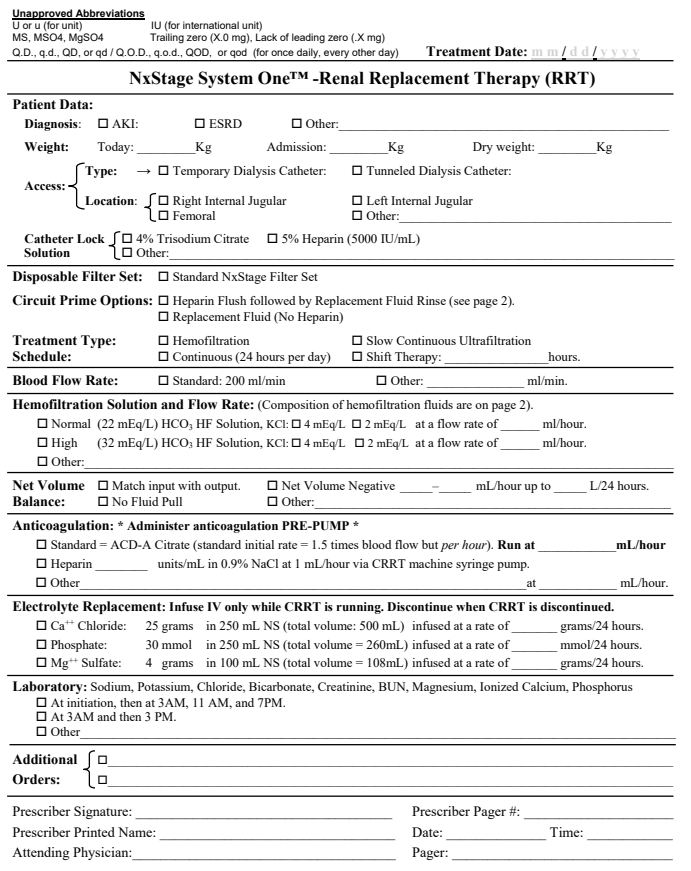

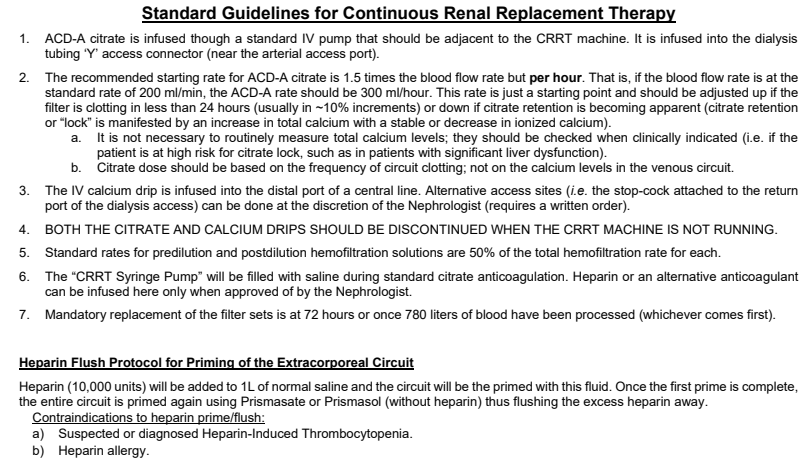

Standard Solutions Used During Continuous Renal Replacement Therapy Standard Citrate Solution:

ACD-A: Anticoagulant Citrate Dextrose Solution Formula A (for infusion as an anticoagulant during CRRT)

$\begin{array}{lrl}\text { Citicic Acid } & 0.73 \mathrm{gm} / 100 \mathrm{ml} \\ \text { Dextrose } & 2.4 \mathrm{gm} / 100 \mathrm{ml} \\ \text { Sodrium Citrate } & 2.20 \mathrm{gm} / 100 \mathrm{ml}\end{array}$

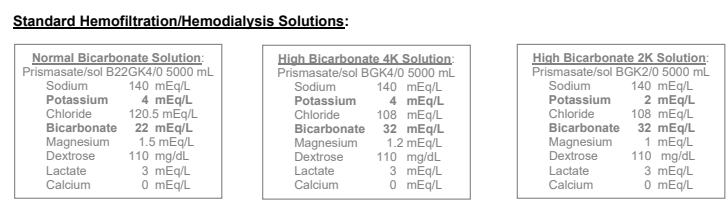

Figure 2. Adult NxStage protocol.

solution with $250 \mathrm{~mL}$ of normal saline [manufactured at a compounding pharmacy]). Following the initial 2448 hours, most patients will need separate infusions of phosphorus (e.g. sodium-phosphate of 30-45 mM/24 h) and magnesium (magnesium chloride of 2-4 gm/24 h). Ionized calcium is not being monitored on the return limb, to minimize expense and avoid potential confusion with regard to very low values (minimizing potential for false alarms and medical errors). For serum ionized calcium, we are targeting normal values $(1.25 \mathrm{mM} / \mathrm{L})$ but with a bias to keep it in a high-normal range (1.10$1.30 \mathrm{mM} / \mathrm{L}$ ) for critically ill patients. Target range for phosphorus is between $3.5-4.5 \mathrm{mg} / \mathrm{dL}$ and $1.5-2 \mathrm{mg} / \mathrm{dL}$ for magnesium. As ACD-A is both a source of bicarbonate 
and hypertonic (sodium $225 \mathrm{mEq} / \mathrm{L}$ ), built-in features of the protocol are the tendencies for metabolic alkalosis and mild hypernatremia, may be advantageous under select circumstances (e.g., brain edema). However, should serum bicarbonate rise to an undesirable degree (e.g., $>28$ $\mathrm{mM} / \mathrm{L}$ ), the blood flow rate can be reduced to $150 \mathrm{~mL} /$ $\mathrm{min}$, with consequential reduction of ACD-A flow rate to 220-230 $\mathrm{mL} / \mathrm{h}$ and decreased net citrate delivery.

For premature filter clotting, at each restart we increase ACD-A rate by $10 \%(30 \mathrm{~mL} / \mathrm{h})$. For hypocalcemia with low ionized calcium, we increase the calcium infusion rate by $10 \%-20 \%$. For "citrate lock" (elevated total calcium with low or normal ionized calcium) we decrease citrate rate by $10 \%$. Should further clotting take place, in the absence of contraindication, we may add fixed low-dose (500-750 units/hour) or aPTT-adjusted I.V. heparin into the circuit. On the other hand, expense is prohibitive for direct thrombin inhibitor argatroban, except in very unusual cases (16). While it is technically possible to add additional potassium to the pre-mixed solution, we generally avoid such practice. In the anecdotal experience of the authors, filter survival usually reached 48-72 hours under these circumstances for most ( 70\%) of the patients.

\section{Additional issues of implementation}

Large volume hemofiltration or dialysate rates ( $>2-3$ $\mathrm{L} / \mathrm{h}$ ) frequently result in hypothermia in the ICU. The use of blood warmers is therefore routinely needed for the extracorporeal circuits with occasional measures of additional passive warming (heating blanket, additional covering of neck-head area). Thyroid dysfunction should always be on the differential for unexplained hypothermia despite these measures (17). For hemodialysis access we are preferentially using double dialysis catheters placed into the internal jugular vein. Patients also need a triple-lumen catheter placed into a central vein for infusion of calcium, phosphate and magnesium. Only in rare circumstances do we permit the omission of triple-lumen catheter placement and the infusion of calcium via the return limb (attending physician's signature required) for those with critical electrolyte abnormalities and central access difficulties, where even a few hours delay would cause harm. Avoiding post-filter ionized calcium monitoring reduced the expense and potential for misunderstanding, reporting on the extremely low, non-physiologic ionized calcium concentrations. Similar abandonment of routine post-filter monitoring has been reported since by others (18) and the accuracy of measured post-filter ionized calcium has been called into question by others, as well $(19,20)$. We routinely place safety locks on the dialysis catheter-to-extracorporeal connection (e.g., HemaClip Bloodline Connector Clip for Hemodialysis, Fresenius Medical Care North America, Waltham, MA) to prevent accidental disconnection (Figure 3). The back page of the protocol serves also as a quick, hands-on reference guide for physicians to review available solutions for renal

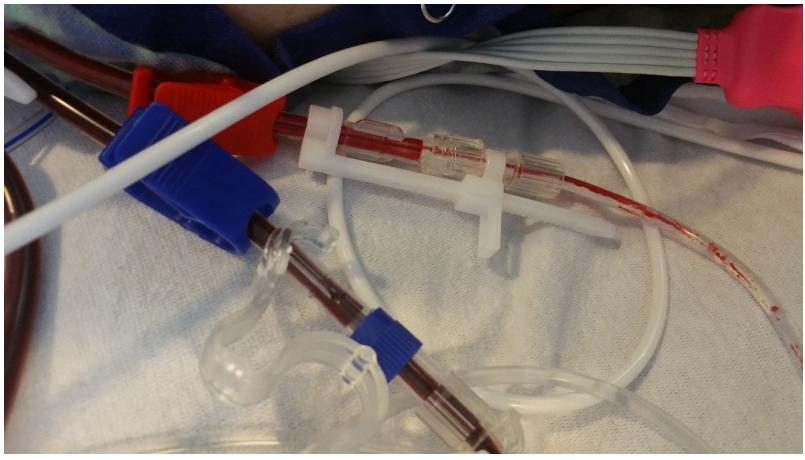

Figure 3. Blood line connector safety clip in place.

replacement therapy. Protocols from Figures 1 and 2 are further adaptable for the individual user's institutions. In our experience, nursing acceptance is critical for successful implementation. Education, including lectures and seminars given to nursing staff, hands-on training session and the development of a cadre of highly trained "super-users" for each ICU is critical. Maintaining training and outcome monitoring is essential and part of ensuring quality of care. Hospital administrations need to be on board, along with the hospital pharmacy program, to recognize the cost effectiveness and full potential of our protocol.

\section{Conclusion}

Abandoning post-filter monitoring of ionized calcium during RCA offers improvement over existing approaches, including simplification of management and reducing the burden of complexity, hence decreasing the potential for medical errors. Our paper described sample protocols of RCA-assisted CRRT and briefly reviewed practical points of implementation. We kept the language of this paper deliberately simple, hence it would be easy to read and understand by non-nephrology physicians, nurses and dialysis technicians alike.

\section{Acknowledgements}

Drs. Fülöp and Zsom are former faculty members of the University of Mississippi Medical Center, Department of Medicine, as well as former Medical Directors of Dialysis at the University of Mississippi Medical Center. Dr. Fülöp is currently affiliated with the Medical University of South Carolina and the Ralph H. Johnson VA Medical Center in Charleston, SC, USA. Dr. Sohail Abdul Salim is a recent graduate of Department of Medicine Nephrology Fellowship at the University of Mississippi Medical Center (Class of 2017); he is currently affiliated with Central Nephrology, Jackson, MS. We sincerely appreciated the assistance of Mr. Attila Lénárt-Muszka during editing and grammar review.

\section{Authors' contribution}

Primary draft by TF. Editing the final manuscript by SAS 
and LZ. All authors read and signed the final manuscript.

\section{Conflicts of interest}

Dr. Zsom is an employee of Fresenius Medical Care (FMC) Hungary and Dr Fülöp is a former employee of FMC Hungary. However, the views and opinions expressed herewith do not reflect the official opinion of the Fresenius Medical Care Hungary.

\section{Ethical considerations}

Ethical issues (including plagiarism, data fabrication, double publication) have been completely observed by the authors.

\section{Funding/Support}

None.

\section{References}

1. Palevsky PM, Zhang JH, O'Connor TZ, Chertow GM, Crowley ST, Choudhury D, et al. Intensity of renal support in critically ill patients with acute kidney injury. $\mathrm{N}$ Engl J Med. 2008;359:7-20. doi: 10.1056/NEJMoa0802639

2. RENAL Replacement Therapy Study Investigators. Intensity of continuous renal - replacement therapy in critically ill patients. N Eng J Med. 2009;361:1627-38. doi: 10.1056/ NEJMoa0902413

3. Fulop T, Pathak MB, Schmidt DW, Lengvarszky Z, Juncos JP, Lebrun CJ, et al. Volume-related weight gain and subsequent mortality in acute renal failure patients treated with continuous renal replacement therapy. ASAIO J. 2010;56:333-7. doi: 10.1097/MAT.0b013e3181de35e4

4. Fülöp T, Zsom L, Tapolyai MB, Molnar MZ, Rosivall L. Volume-related weight gain as an independent indication for renal replacement therapy in the intensive care units. J Renal Inj Prev. 2017;6:35-42. doi: 10.15171/jrip.2017.07

5. Zhang Z, Ni H, Fan H, Li D, Xu X. Actually delivered dose of continuous renal replacement therapy is underestimated in hemofiltration. ASAIO J. 2013;59:622-6. doi: 10.1097/ MAT.0000436713.34635.a8

6. Greinacher A. Heparin-induced thrombocytopenia. New Engl J Med. 2015;373:252-61. doi: 10.1056/NEJMcp1411910

7. Warkentin TE. Heparin-induced thrombocytopenia. Curr Opin Crit Care. 2015;21:576-85. doi: 10.1097/ MCC.0000000000000259

8. Greinacher A, Selleng K. Thrombocytopenia in the intensive care unit patient. Hematology Am Soc Hematol Educ Program. 2010;2010:135-43. doi: 10.1182/ asheducation-2010.1.135

9. Strauss R, Wehler M, Mehler K, Kreutzer D, Koebnick C, Hahn EG. Thrombocytopenia in patients in the medical intensive care unit: bleeding prevalence, transfusion requirements, and outcome. Crit Care Med.
2002;30:1765-71

10. Flanigan MJ, Pillsbury L, Sadewasser G, Lim VS. Regional hemodialysis anticoagulation: hypertonic tri-sodium citrate or anticoagulant citrate dextrose-A. Am J Kidney Dis. 1996;27:519-24. doi: 10.1016/S0272-6386(96)90162-6

11. Palsson R, Niles JL. Regional citrate anticoagulation in continuous venovenous hemofiltration in critically ill patients with a high risk of bleeding. Kidney Int. 1999;55:1991-7. doi: 10.1046/j.1523-1755.1999.00444.x

12. Yessayan LT, Yee J, Frinak S, Szamosfalvi B. Online Hemoglobin and Oxygen Saturation Sensing During Continuous Renal Replacement Therapy with Regional Citrate Anticoagulation. ASAIO J. 2015;61:489-95. doi: 10.1097/MAT.0000000000000057

13. Kutsogiannis DJ, Gibney RT, Stollery D, Gao J. Regional citrate versus systemic heparin anticoagulation for continuous renal replacement in critically ill patients. Kidney Int. 2005;67:2361-7. doi: 10.1111/j.15231755.2005.00342.x

14. Morabito S, Pistolesi V, Tritapepe L, Fiaccadori E. Regional citrate anticoagulation for RRTs in critically ill patients with AKI. Clin J Am Soc Nephrol. 2014;9:2173-88. doi: 10.2215/ CJN.01280214

15. Schilder L, Nurmohamed SA, ter Wee PM, Paauw NJ, Girbes AR, Beishuizen A, et al. Citrate confers less filter-induced complement activation and neutrophil degranulation than heparin when used for anticoagulation during continuous venovenous haemofiltration in critically ill patients. BMC Nephrol. 2014;15:19. doi: 10.1186/1471-2369-15-19

16. Ferguson LM, Dreisbach AW, Csongradi E, Juncos LA, Fulop T. Recurring extracorporeal circuit clotting during continuous renal replacement therapy in fungal sepsis: successful treatment with argatroban. Am J Med Sci. 2013;345:256-8. doi: 10.1097/MAJ.0b013e3182711e59

17. Kumar E, McCurdy MT, Koch CA, Hamadah A, Fülöp T, Gharaibeh KA. Functional Impairment of Thyroid Function in Critically Ill Patients in the Intensive Care Units. Am J Med Sci. 2017. doi: 10.1016/j.amjms.2017.06.026

18. Schilder L, Nurmohamed SA, Bosch FH, Purmer IM, den Boer SS, Kleppe CG, et al. Citrate anticoagulation versus systemic heparinisation in continuous venovenous hemofiltration in critically ill patients with acute kidney injury: a multi-center randomized clinical trial. Crit Care. 2014;18:472. doi: 10.1186/s13054-014-0472-6

19. Schwarzer P, Kuhn S-O, Stracke S, Gründling M, Knigge S, Selleng S, et al. Discrepant post filter ionized calcium concentrations by common blood gas analyzers in CRRT using regional citrate anticoagulation. Critical Care. 2015;19:321. doi: 10.1186/s13054-015-1027-1

20. Oudemans-van Straaten HM, Ostermann M. Citrate anticoagulation for CRRT: don't always trust the postfilter iCa results! Critical Care. 2015;19:429. doi: 10.1186/s13054015-1148-6

Copyright (c) 2018 The Author(s); Published by Nickan Research Institute. This is an open-access article distributed under the terms of the Creative Commons Attribution License (http://creativecommons.org/licenses/by/4.0), which permits unrestricted use, distribution, and reproduction in any medium, provided the original work is properly cited. 